\title{
The Brassica rapa Rubber Elongation Factor Promoter Regulates Gene Expression During Seedling Growth in Arabidopsis thaliana and Brassica napus
}

\author{
Joon Ki Hong, Myung-Ho Lim, Jin A Kim, Jung Sun Kim, Seung Bum Lee, Eun Jung Suh, Soo In Lee, Yeon-Hee Lee* \\ National Academy of Agricultural Science, Rural Development Administration, 370 Nongsaengmyeong-ro, Jeonju-si, Jeollabuk-do, Korea
}

\begin{abstract}
A tissue-specific and developmentally expressed gene was isolated from Chinese cabbage (Brassica rapa L. ssp. pekinensis), designated BrREF (B. rapa Rubber elongation factor). BrREF transcripts were expressed at high levels in seedlings and at low levels in flower buds and roots. To study the activity of this promoter, the $2.2 \mathrm{~kb}$ upstream sequence of $B r R E F$ gene was fused to a $\beta$-glucuronidase (GUS) reporter gene and was introduced into Arabidopsis thaliana and B. napus by Agrobacterium-mediated transformation. Strong expression of GUS driven by the BrREF promoter was detected in the cotyledons and hypocotyls of transgenic plant seedlings, but GUS expression was weak in roots, excluding the root tips. GUS expression in the cotyledons and hypocotyls decreased dramatically as the seedlings matured and was not detected in the tissues of mature plants. During floral development, GUS expression was observed in immature anthers. These findings suggest that the BrREF promoter can modulate the tissue-specific and developmental expression of gene at the early stages of growth and development.
\end{abstract}

Keywords Chinese cabbage, GUS, Promoter, Rubber elongation factor, Transgenic plant

\section{INTRODUCTION}

Rubber elongation factor (REF) plays a major role in rubber polymerization and is located on the surface of large rubber particles in latex (Dennis et al. 1989; Dennis and Light 1989; Priya et al. 2006). Rubber biosynthesis takes place on the surface of rubber particles suspended in latex produced in laticifers, which are specialized secretory cells (Dennis and Light 1989). REF gene expression is also highly induced in laticifers (Dennis et al. 1989). The amount of REF protein in whole latex is proportional to the rubber content. The presence of REF, which is associated with large rubber particles in latex, is required for rubber molecule elongation. Thus, REF is likely to be an important enzymatic component of rubber biosynthesis. Interestingly, in addition to genes involved in rubber biosynthesis, genes induced by wounding and ethylene responses are expressed at high levels in laticifers (Han et al. 2000; Kush et al. 1990).
Recently, the identification and characterization of genomic DNA from Hevea brasiliensis encoding the REF gene and its promoter sequence were reported (Priya et al. 2006, 2007). HbREF transcripts were abundant in latex cells of high yielding rubber clones, indicating that a high level of $R E F$ transcript accumulation in high yielding clones correlated closely with latex yield in $H$. brasiliensis (Priya et al. 2007). In addition, GUS expression driven by the $H b R E F$ promoter in transgenic Arabidopsis was observed in the vascular tissues of leaves, stems, and midribs and was also induced by wounding. It was suggested that $R E F$ expression was regulated in a tissue-specific manner and was induced by environmental cues related to wounding (Priya et al. 2006). Despite the important role of REF in rubber biosynthesis, the tissue-specific expression of the $R E F$ gene and its regulation in response to developmental and environmental cues have not been examined fully in plants other than $H$. brasiliensis.

Currently, there is no report on isolation and characterization

Received July 29, 2014; Revised August 28, 2014; Accepted August 31, 2014; Published September 30, 2014

*Corresponding author Yeon-Hee Lee, yhl2222@korea.kr, Tel: +82-63-238-4651, Fax: +82-63-238-4654 
of a $R E F$ gene from Brassica rapa ( . . rapa rubber elongation factor; $B r R E F)$. To determine the expression mechanism of $B r R E F$ gene during plant development, we cloned the $B r R E F$ promoter and studied the spatial and temporal distribution of its activity in transgenic Arabidopsis and $B$. napus. Our results show that the $B r R E F$ promoter confers specific spatial-temporal GUS reporter gene expression during seedling growth and in floral organs of transgenic Arabidopsis and B. napus, suggesting that this promoter leads to tissue-specific and developmental expression of target genes in heterologous transgenic plants.

\section{MATERIALS AND METHODS}

\section{Plant materials and growth conditions}

Surface-sterilized seeds of $B$. rapa L. ssp. pekinensis and the winter-type B. napus cultivar "Youngsan" were placed on MS medium (Murashige and Skoog 1962) containing $3 \%$ sucrose and $0.8 \%$ Phytagar (pH 5.8) and stratified at $4{ }^{\circ} \mathrm{C}$ for five days in dark condition to induce synchronous germination. The plants were grown at $25^{\circ} \mathrm{C}$ for two weeks

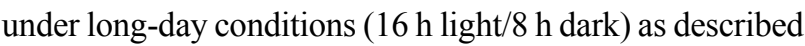
by Yang et al. (2006) and then transplanted to horticultural substrate. The plants were transferred to a growth chamber, and the samples were collected throughout the sampling period.

A. thaliana ecotype Columbia (Col-0) was used as the wild type. The Col-0 seeds were surface-sterilized by vapor-phase sterilization (http://www.plantpath.wisc.edu/ fac/afb/vapster.html; Desfeux et al. 2000), placed on MS medium supplemented with vitamins, $1 \%$ sucrose, and $0.3 \%$ phytagel (Sigma, St. Louis, MO, USA), and stratified at $4^{\circ} \mathrm{C}$ for three days in dark condition to induce synchronous germination. The stratified seeds were germinated under a $16 \mathrm{~h}$ light $/ 8 \mathrm{~h}$ dark photoperiod. Plants were transferred to soil and grown at $23^{\circ} \mathrm{C}$ under $16 \mathrm{~h}$ light $/ 8 \mathrm{~h}$ dark conditions for transformation. Samples were collected throughout the growth period.

\section{Cloning and characterization of the BrREF gene}

Publicly available genome databases were used to search for $B$. rapa REF gene sequences. The DNA sequence of the
Arabidopsis REF (At2g47780, accession no. NM_130345) gene was obtained from The Arabidopsis Information Resource database (http://www.arabidopsis.org) and used to search for $B$. rapa homologs from the sequence database of bacterial artificial chromosome (BAC) clones of B. rapa L. ssp. pekinensis cv. Chiifu using tblastx at NCBI (http:// blast.ncbi.nlm.nih.gov/Blast.cgi). Exon-intron splice sites were analyzed and confirmed by comparing the $B$. rapa BAC clone (KBrB042E01, accession no. AC232484) and the BrREF cDNA sequence using GENSCAN software (http://genes.mit.edu/GENSCAN.html; Burge and Karlin 1998) and GeneMark software (http://opal.biology.gatech. edu/GeneMark/; Lukashin and Borodovsky 1998). Other deduced REF amino acid sequences derived from cDNA sequences were obtained from NCBI (http://www.ncbi. nlm.nih.gov). Analyses of DNA and deduced amino acid sequences were performed using current bioinformatics tools. Amino acid sequence alignment was performed using the GeneDoc program (http://www.pcs.edu/biomed/ genedoc). Pairwise sequence identity values were calculated using MEGALIGN software (DNASTAR, Madison, USA) and the CLUSTAL W algorithm with a PAM250 residue weight table.

\section{Semi-quantitative RT-PCR analysis}

To analyze $B r R E F$ expression in B. rapa, total RNA was extracted from $B$. rapa seedlings and tissues using Trizol reagent (Invitrogen, Carlsbad, CA, USA). For semiquantitative RT-PCR analysis, cDNA was synthesized from $2 \mu \mathrm{g}$ total RNA using MMLV reverse transcriptase (RNaseH free; Toyobo, Osaka, Japan) according to the manufacturer's instructions. Each cDNA sample was diluted 1:3, and $1 \mu 1$ diluted cDNA together with a genespecific primer set (Supplemental Table 1) were used for PCR amplification $\left(94^{\circ} \mathrm{C}\right.$ for $30 \mathrm{~s}, 55^{\circ} \mathrm{C}$ for $30 \mathrm{~s}, 72^{\circ} \mathrm{C}$ for $45 \mathrm{~s}$ ) in a $25 \mu \mathrm{l}$ reaction volume. PCR reactions contained $1 \times$ PCR buffer, $1.5 \mathrm{mM} \mathrm{MgCl}_{2}, 0.5 \mu \mathrm{M}$ of each primer, 0.2 $\mathrm{mM}$ of each dNTP, and 2.5 units Taq DNA polymerase (Takara, Shiga, Japan). The Bactin gene was used as the internal control for RNA quantification (Hong et al. 2013; Yao et al. 2005). After a standard PCR of 30 cycles, the resulting PCR products (5 $\mu \mathrm{l}$ each) were analyzed using electrophoresis and ethidium bromide staining. All PCR 
products were sequenced and matched to individual gene sequences (data not shown). The relative expression levels were analyzed by RT-PCR amplification using the Quantity One program (Bio-Rad, Hercules, CA, USA; Liang et al. 2009, 2011).

\section{Construction of $B r R E F$ promoter-GUS expression vectors}

The DNA sequence upstream of the translation start site $(B r R E F$ promoter, -2176 to +1$)$ was retrieved from a BAC clone sequence. For promoter analysis in transgenic plants, the 2.2-kb 5' upstream promoter region of the $B r R E F$ gene was amplified by PCR from a BAC clone using EX-Taq polymerase (Takara, Shiga, Japan). The PCR amplicon was cloned into the pGEM-T Easy vector (Promega, Madison, WI, USA) and sequenced to confirm the fidelity of amplification. The promoter sequence was analyzed using the plantCARE (http://bioinformatics.psb.ugent.be/ webtools/plantcare/html/) and PLACE (http://www.dna. affrc.go.jp/PLACE/) databases (Higo et al. 1999; Rombauts et al. 1999). The BrREF promoter was digested with Hind III and $E c o$ RI and subcloned into the same restriction sites of the pCAMBIA1391 vector. The construct was transformed into Agrobacterium tumefaciens strain GV3101 containing the pSOUP vector (Hellens et al. 2000) by the freeze-thaw shock method (Holsters et al. 1978).

\section{Generation of transgenic Arabidopsis and B. napus plants}

The binary vector containing the $B r R E F$ promoter-GUS expression construct was introduced stably into Arabidopsis plants using the Agrobacterium tumefaciens-mediated spray method (Hong et al. 2012; Kim et al. 2007). Flowers of Arabidopsis were sprayed with A. tumefaciens GV3101 containing binary vectors suspended in 5\% sucrose and $0.05 \%$ Silwet-L77, and the plants were then incubated in a growth chamber at $23^{\circ} \mathrm{C}$ and $100 \%$ humidity for one day and then grown in a growth chamber under a $16 \mathrm{~h}$ light $/ 8 \mathrm{~h}$ dark photoperiod at $23^{\circ} \mathrm{C} . \mathrm{T}_{1}$ seeds and progeny were germinated on MS medium containing $30 \mathrm{mg} / \mathrm{L}$ hygromycin for the selection of transformants. $T_{3}$ generation transgenic plants were selected for analysis.

The B. napus transformation method used was similar to that of Hong et al. (2013). Surface-sterilized seeds of the winter-type B. napus cultivar "Youngsan" were planted on MS medium containing 3\% sucrose and $0.8 \%$ Phytagar and incubated in a culture room under a $16 \mathrm{~h}$ photoperiod at $25^{\circ} \mathrm{C}$ for six to seven days. Hypocotyls were cut into $0.5 \mathrm{~cm}$ segments and placed on pre-culture medium (MS basal medium, $1 \mathrm{mg} / \mathrm{L}$ NAA, $2 \mathrm{mg} / \mathrm{L} \mathrm{BA}, 2 \mathrm{mg} / \mathrm{L} \mathrm{AgNO}_{3}$, 3\% sucrose, and $0.8 \%$ Phytagar) for two days. The pre-cultured hypocotyls were inoculated with Agrobacterium suspension $\left(\mathrm{OD}_{600}=0.3\right)$ in MS liquid medium for 15-20 min with gentle shaking. The inoculated hypocotyls were blotted with sterilized filter paper to remove excess bacterial suspension and cocultivated on cocultivation medium (MS basal medium, $1 \mathrm{mg} / \mathrm{L} \mathrm{NAA}, 2 \mathrm{mg} / \mathrm{L} \mathrm{BA}, 2 \mathrm{mg} / \mathrm{L} \mathrm{AgNO}_{3}$, $100 \mu \mathrm{M}$ acetosyringone, $3 \%$ sucrose, and $0.8 \%$ Phytagar) for three days in darkness at $25^{\circ} \mathrm{C}$. Hypocotyls were washed with sterilized water containing $100 \mathrm{mg} / \mathrm{L}$ carbenicillin and $250 \mathrm{mg} / \mathrm{L}$ cefotaxime and transferred to selection medium (MS basal medium, $1 \mathrm{mg} / \mathrm{L} \mathrm{NAA,} 2 \mathrm{mg} / \mathrm{L} \mathrm{BA}, 2 \mathrm{mg} / \mathrm{L}$ $\mathrm{AgNO}_{3}, 3 \%$ sucrose, $100 \mathrm{mg} / \mathrm{L}$ carbenicillin, $250 \mathrm{mg} / \mathrm{L}$ cefotaxime, and $0.8 \%$ Phytagar) supplemented with 15 $\mathrm{mg} / \mathrm{L}$ hygromycin as a selection agent. Callus that formed on hypocotyls was subcultured on fresh selection medium at two- or three-week intervals. Healthy and elongated shoots were excised and transferred to phytohormone-free MS medium to induce rooting. The transformed plants were grown in a greenhouse after acclimatization. $T_{2}$ generation transgenic plants were selected for analysis.

\section{Verification of transgenic Arabidopsis and B. napus}

To confirm the presence of the transgenes in transgenic plants, we performed PCR analysis of genomic DNA using a $G U S$-specific primer set (Supplemental Table 1). The PCRs were performed using 30 cycles of $30 \mathrm{~s}$ at $95^{\circ} \mathrm{C}, 30 \mathrm{~s}$ at $55^{\circ} \mathrm{C}$, and $45 \mathrm{~s}$ at $72^{\circ} \mathrm{C}$, followed by a final extension for 10 min at $72^{\circ} \mathrm{C}$.

To analyze the expression of the GUS gene using semi-quantitative RT-PCR, total RNA was extracted from ten-day-old transgenic plants. Two micrograms of total RNA were converted into cDNA as described above (Section "Semi-quantitative RT-PCR analysis"). Each cDNA was diluted 1:3, and $1 \mu \mathrm{l}$ cDNA was used for PCR amplification with a GUS-specific primer set (Supplemental 
Table 1). PCR amplification of the AtActin and Bactin gene was performed as a quantitative control. Aliquots of individual PCR products were resolved by agarose gel electrophoresis and visualized by ethidium bromide under UV light.

\section{Histochemical analysis of GUS activity}

Histochemical assays for GUS activity were performed on various tissues from transgenic plants at various developmental stages using 5-bromo-4-chloro-3-indolyl- $\beta$ -d-glucuroide (X-gluc; Duchefa, Haarlem, The Netherlands) as a substrate (Jefferson et al. 1987). Tissues were stained at $37^{\circ} \mathrm{C}$ in X-gluc reaction buffer $(50 \mathrm{mM}$ sodium phosphate buffer, $\mathrm{pH} 7.2,0.05 \%$ Triton $\mathrm{X}-100,1 \mathrm{mM}$ potassium ferrocyanide and potassium ferricyanide, and $2 \mathrm{mMX}$-gluc) for $24 \mathrm{~h}$. Subsequently, the samples were transferred to $70 \%$ ethanol to remove chlorophyll and stored in $80 \%$ ethanol at $4{ }^{\circ} \mathrm{C}$ before imaging. The GUS-positive plant tissues were examined under a bright field microscope (Olympus SZX12; Olympus, Japan). The GUS-stained tissue and plant images presented here represent typical results from at least three independent lines per construct.

\section{RESULTS}

\section{Identification and characterization of the $R E F$ gene from $B$. rapa}

A search was performed for $B r R E F$ gene sequences in publicly available genome databases. To assemble the $B r R E F$ gene sequence, we used in silico approach to screen the B. rapa genome (Hong et al. 2010; Zhang et al. 2009). A full-length AtREF (At2g47780) gene sequence containing the conserved REF protein sequence was blasted against a sequenced BAC clone derived from B. rapa L. ssp. pekinensis $\mathrm{cv}$. Chiifu using tblastx in the NCBI B. rapa database. The selected BAC clone was annotated using GENSCAN and GeneMark software. A BrREF sequence was found in the sequence of a BAC clone (KBrB042E01), from which the predicted $B r R E F$ gene was excised for further analysis. The nucleotide sequence of the $B r R E F$ (KJ489412) gene was determined and presented in Fig. 1. Sequence analysis of the identified $R E F$ genomic DNA revealed that it comprises 800 base pairs. Comparison of the cDNA sequence and the BAC clone sequence revealed that the $B r R E F$ gene consists of two exons separated by a 78-bp intron, which was identical to the cDNA sequence.

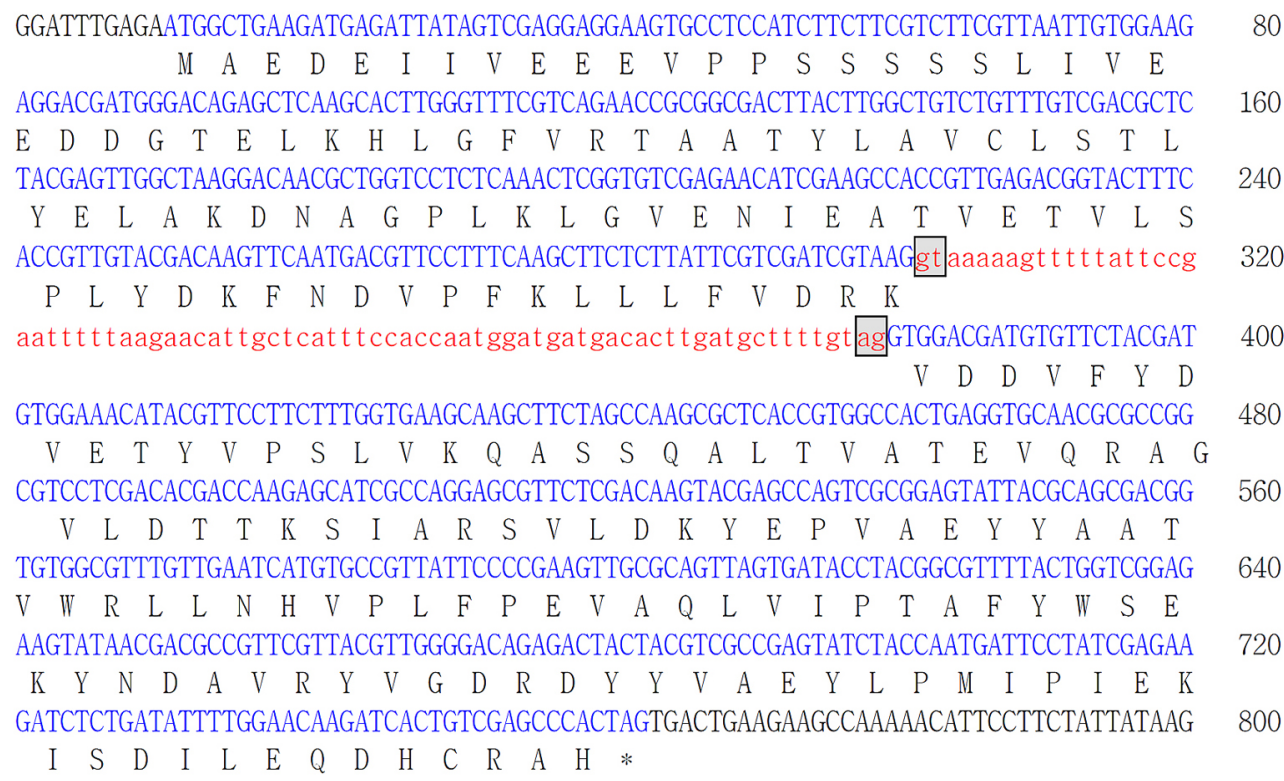

Fig. 1. Nucleotide and deduced amino acid sequence of genomic DNA encoding $B r R E F$. The blue uppercase letters represent exons. The red lowercase letters represent introns and splice donor (GT) and accept (AG) were boxed. The deduced amino acid sequences are presented by a single letter code below the exon sequences. The stop codon is marked with an asterisk. 
The $B r R E F$ gene contained a 675-bp open reading frame and encoded a putative protein of 224 amino acids with a calculated molecular mass of $25 \mathrm{kDa}$.

To determine the relationships between the BrREF protein and other REF-related proteins, we performed an alignment of the deduced amino acid sequence of BrREF with those of REF-related proteins from other species (Fig. $2)$. The pairwise identity among the deduced REF protein amino acid sequences varied from 45.4 to $85.3 \%$ indicating that the REF-related proteins are quite divergent in amino acid sequence (Supplemental Table 2). Despite this, the predicted BrREF protein was closely related to the

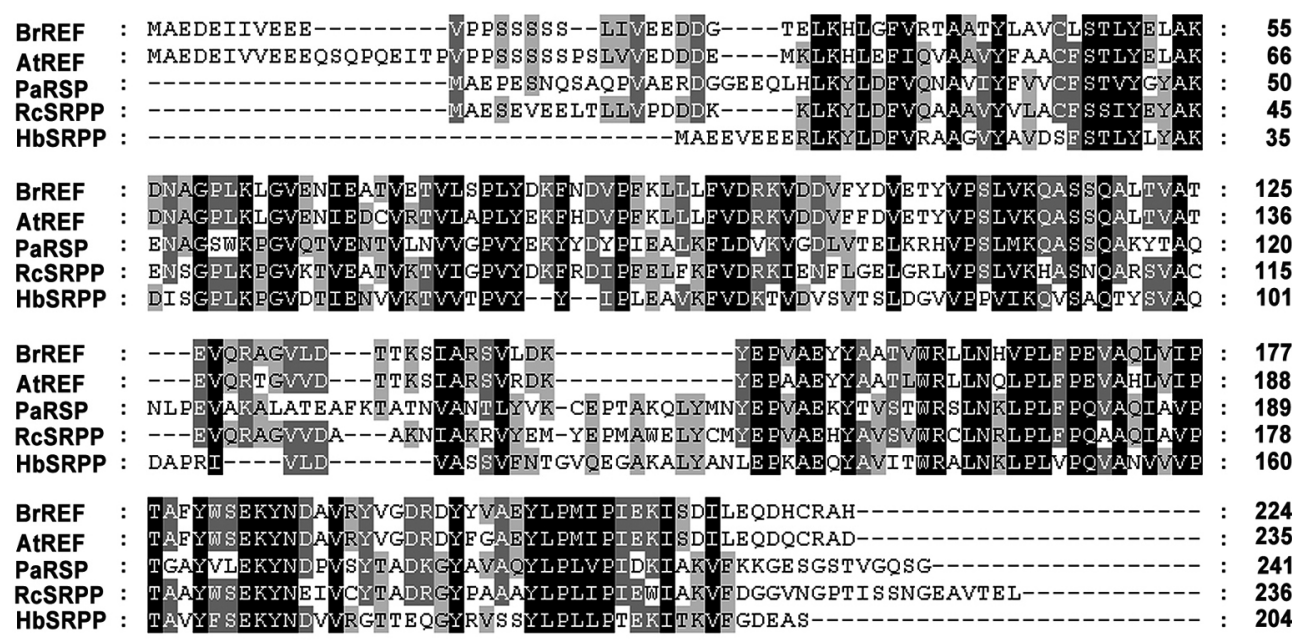

Fig. 2. Comparison of the amino acid sequences of the REF proteins. Alignment of the deduced amino acid sequences of BrREF with AtREF and REF-related protein (SRPP) from other plant species is shown. Accession numbers of sources from GenBank database are as follows: Arabidopsis thaliana, AtREF (NP_182299); Brassica rapa, BrREF (KJ489412); Hevea brasiliensis, HbSRPP (AAC82355); Parthenium argentatum, PaRSP (AAQ11374); Ricinus communis, RcSRPP (EEF30521).

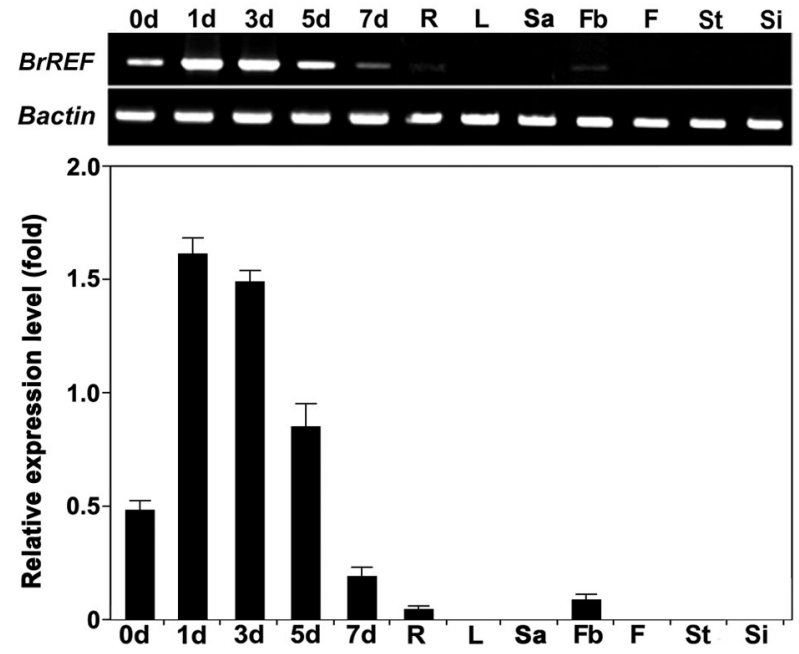

Fig. 3. $B r R E F$ expression during seedling growth and in various tissues of $B$. rapa. Total RNA was isolated from seedlings (lanes 0d-7d), roots (lane R), leaves (lane L), shoot apical meristems (lane Sa), flower buds (lane Fb), flowers (lane F), stems ( $\mathrm{St}$ ), and siliques ( $\mathrm{Si}$ ). A 2- $\mu \mathrm{g}$ aliquot of total RNA was reverse-transcribed to produce first-strand cDNA for semi-quantitative RT-PCR analysis of gene expression. The Bactin gene was used as an internal control for RNA quantification. Data are presented as means \pm standard deviation (SD) from at least three independent experiments. 
Arabidopsis REF protein, indicating that the Arabidopsis and $B$. rapa proteins were probably functionally homologous. However, the BrREF and AtREF protein sequences were very different from orthologous proteins in other species. This observation suggests that the cellular function of the REF protein of B. rapa may differ from those of REFrelated proteins from other species and may not be functionally equivalent.

To define the spatio-temporal pattern of $R E F$ gene expression in $B$. rapa, we analyzed $B r R E F$ expression in various tissues during different growth stages using semiquantitative RT-PCR. BrREF was expressed strongly during the early stages of seedling growth, while expression was low during the late stages of seedling development and in roots and flower buds (Fig. 3). However, BrREF expression was not detected in mature leaves, flowers, stems, or siliques.

\section{Characterization and analysis of $B r R E F$ promoter-GUS transgenic Arabidopsis and B. napus plants}

To analyze the specific expression mechanism of $B r R E F$, we fused the putative promoter region (2.2 kb 5 ' upstream region) of $B r R E F$ to a GUS reporter gene (Supplemental Fig. 1A) and introduced the construct into Arabidopsis and B. napus by Agrobacterium-mediated transformation (Hong et al. 2013; Kim et al. 2007). From the $\mathrm{T}_{1}$ plants, we selected 20 independent lines on selection medium containing hygromycin. From the 20 transgenic plants, three independent transgenic plants that exhibited a progeny segregation ratio of $3: 1$ for resistance trait consistent with expected Mendelian inheritance of one independent locus were selected for further analysis. PCR analysis confirmed the presence of the GUS gene in each of the selected plants (Supplemental Fig. 1B). GUS gene expression was examined in $\mathrm{T}_{2}$ transgenic plants by $G U S$ gene-specific RT-PCR using total RNA from transgenic

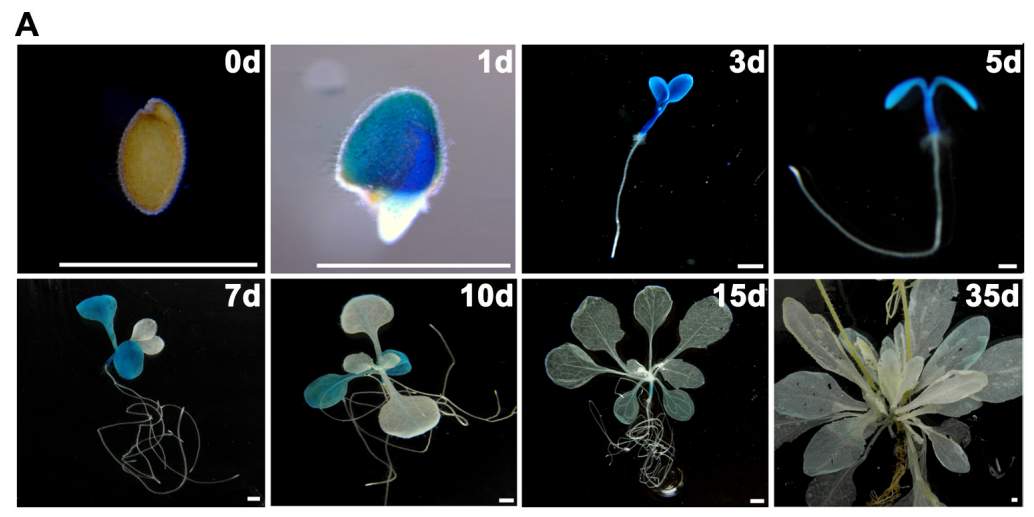

B

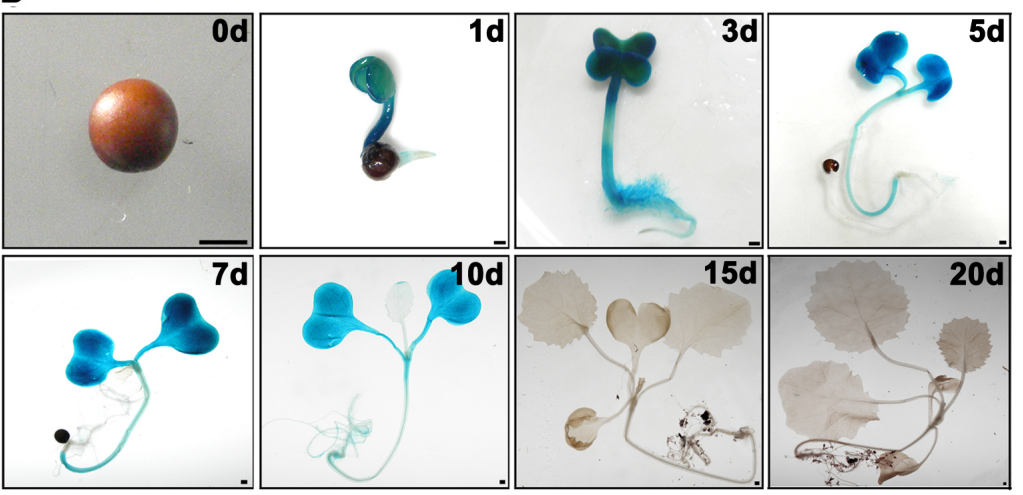

Fig. 4. Promoter activity in $B r R E F$ promoter-GUS transgenic plants measured over a series of developmental stages. (A) GUS expression in BrREF promoter-GUS transgenic Arabidopsis. (B) GUS expression in BrREF promoter-GUS transgenic B. napus. Bars are $1 \mathrm{~mm}$. 
seedlings. GUS transcripts were expressed at high levels in transgenic seedlings relative to wild-type (Supplemental Fig. 1C), indicating that GUS expression in the seedlings was conferred by the BrREF promoter.

Furthermore, to examine the specific expression patterns of the REF promoter, GUS activity was monitored throughout plant growth using a histochemical procedure. GUS activity in a developmental series of transgenic Arabidopsis and B. napus plants is shown in Fig. 4. GUS expression was detected during seedling growth of $B r R E F$ promoter-GUS transgenic Arabidopsis and B. napus plants. In transgenic Arabidopsis, high GUS expression was detected in the cotyledons and hypocotyls of germinating seeds and during seedling growth, while low GUS expression was detected in roots, excluding the root tips. However, GUS expression decreased dramatically after ten days and was detected after 15 days only in the hypocotyl region (Fig. 4A and 5A). In transgenic B. napus, the pattern of GUS expression driven by the $B r R E F$ promoter was very similar to that of transgenic Arabidopsis with high expression in the cotyledons and hypocotyls during seedling growth (Fig. 4B). This suggests that the BrREF promoter was responsible for the precise transcription regulation determining the unique spatial and temporal expressions of the BrREF gene in seedling and anther growth of the transgenic plants. Interestingly, GUS expression driven by the $B r R E F$ promoter was almost absent during the mature plant stages. Thus, GUS activities detected in cotyledons and hypocotyls during seedling growth were similar in BrREF promoter-GUS transgenic Arabidopsis and $B$. napus plants (Fig. 4A and B).

To gain insight into $B r R E F$ promoter activity during the reproductive stage, we examined $B r R E F$ promoter-driven patterns of GUS expression in the reproductive organs and tissues (Fig. 5B and C). In transgenic Arabidopsis, GUS expression driven by the $B r R E F$ promoter was observed only in the immature styles, septums, and anthers of flower buds, but it was not detected in open flowers (Fig. 5B) or other reproductive tissues (data not shown). However, the GUS expression in the floral organs of transgenic B. napus showed spatial and temporal differences. GUS was expressed constitutively in the anthers of flower buds and open flowers and expressed at low levels in the stigma of flower buds (Fig. 5C).

To precisely determine the developmental series and tissue-specificity of the $B r R E F$ promoter in transgenic Arabidopsis and B. napus plants, semi-quantitative RT-PCR was performed. RT-PCR analysis revealed that $G U S$ gene
A

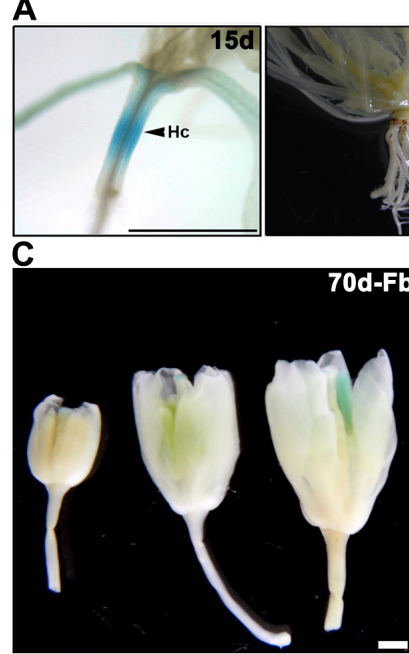

B

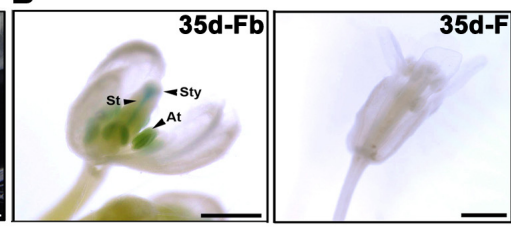

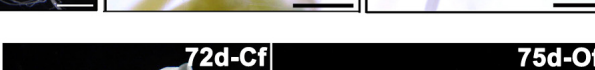

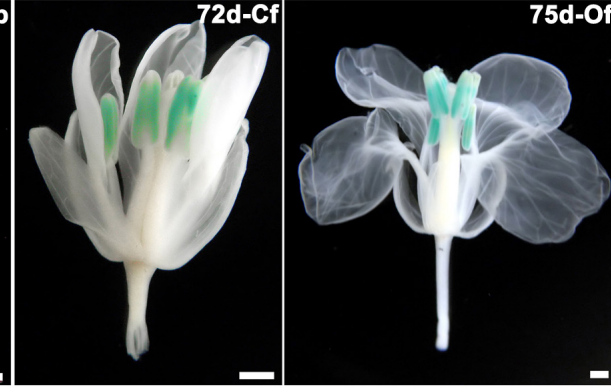

Fig. 5. Histochemical analysis of GUS expression driven by the $B r R E F$ promoter in different organs of transgenic plants. (A) Promoter activity in the hypocotyls of BrREF promoter-GUS transgenic Arabidopsis plants. Bars are $1 \mathrm{~mm}$. (B) GUS expression in the floral organs of BrREF promoter-GUS transgenic Arabidopsis plants. Bars are 200 $\mu \mathrm{m}$. (C) GUS expression in the floral organs of BrREF promoter-GUS transgenic B. napus plants. Bars are $1 \mathrm{~mm}$. At, anther; Cf, Closed flower; F, flower; Fb, Flower bud; Hc, hypocotyl; Of, open flower; St, septum; Sty, style. 
transcripts were expressed at high levels in seedlings and at low levels in flower buds, although their intensities varied (Fig. 6).

\section{Sequence analysis of the BrREF promoter}

To identify cis-acting elements in the putative $B r R E F$ promoter, we analyzed the BrREF promoter sequence using the PLACE (Higo et al. 1999) and PlantCARE (Rombauts et al. 1999) databases. The nucleotide sequence of the $B r R E F$ promoter region and putative regulatory motifs are shown in Fig. 7. The BrREF promoter contains the TATA and CAAT box elements shown to be necessary for an accurate initiation of basal transcription in promoters of other plants. In addition, we identified potential regulatory elements associated with growth- and phytohormone-related response including an abscisic acid-responsive element (ABRE), an auxin-responsive element (AuxRR-core), an ABA- and VP1-responsive element (CE3), a cis-acting element conferring high transcription levels (5'UTR-Py-rich), a cis-acting element required for endosperm expression (GCN4-motif, Skn-1 motif), and a gibberellin-responsive element (GARE). Furthermore, this promoter contains several putative defense and stress-responsive transcription factor-binding sites, including the following: an enhancement of anoxic-specific inducibility motif (GC-motif), a fungal elicitor-responsive element (Box-W1), a MeJA-responsive motif (TGACG motif), a MYB binding site involved in drought inducibility (MBS), a salicylic acid-responsive element (TCA-element), and a wound-responsive element (WUN-motif). Based on these findings, these elements may modulate gene expression in tissue-specific and developmental expression at the early stages of growth and development.

\section{DISCUSSION}

The BrREF gene in Chinese cabbage codes for a putative rubber elongation factor which involved in rubber biosynthesis pathway. In a previous study, the expression of $R E F$ gene in $H$. brasiliensis was detected in all tissues except roots and was expressed at high levels in lacticiferous phloem

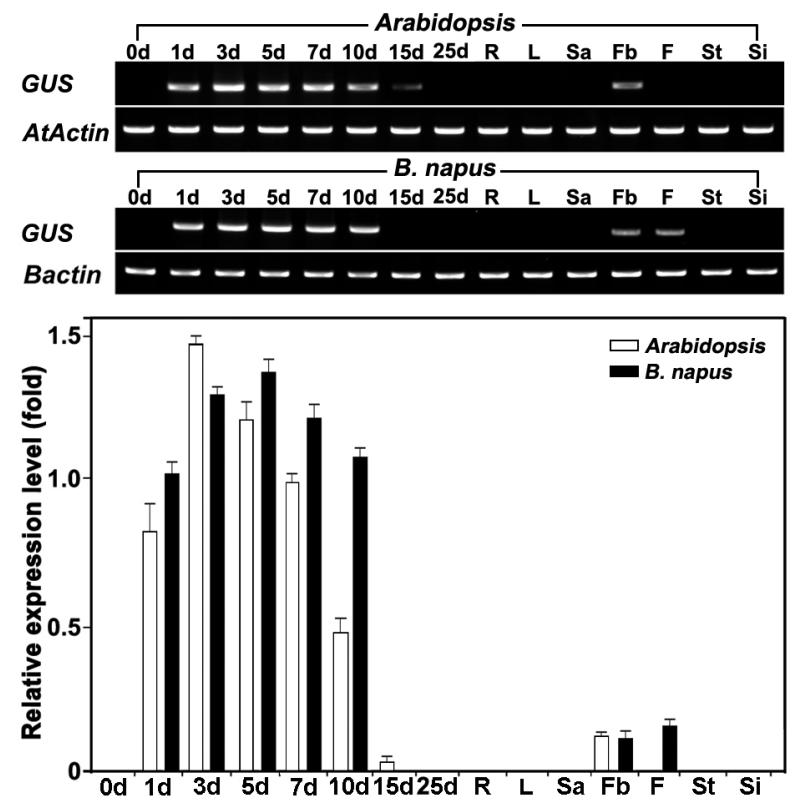

Fig. 6. GUS gene expression during seedling growth and in various tissues of transgenic Arabidopsis and B. napus. Total RNA was isolated from seedlings (lanes 0d-10d), vegetative growth stages (lanes $15 \mathrm{~d}-25 \mathrm{~d}$ ), roots (lane R), leaves (lane L), shoot apical meristems (lane Sa), flower buds (lane Fb), flowers (lane F), stems ( $\mathrm{St}$ ), and siliques ( $\mathrm{Si}$ ). A 2- $\mu \mathrm{g}$ aliquot of total RNA was reverse-transcribed into first-strand cDNA for semi-quantitative RT-PCR analysis of gene expression. AtActin and Bactin gene expression were used as a quantitative control. Data are means \pm standard deviation (SD) from at least three independent experiments. 
tissues (Priya et al. 2006, 2007). Also, the $H b R E F$ promoter was shown to confer strong GUS expression in the vascular tissues of leaves, stems, and midribs of transgenic Arabidopsis. In this study, the $B r R E F$ gene was expressed at the early stages during seedling growth and floral organs in B. rapa, indicating that this gene expression pattern was different from that of $H b R E F$ (Fig. 3). This observation suggests that the $B r R E F$ gene may play a role in the early growth of seedlings, even though it was also expressed in the late seedling stages and other tissues at variable levels. The selective induction of $B r R E F$ gene expression may be due to the presence of specific promoter elements (Hwang et al. 2009).

Promoter sequences are predicted to drive gene expression in a specific spatio-temporal manner during plant growth and development (Hong et al. 2011). To investigate the

\begin{tabular}{|c|c|c|}
\hline-2176 & $\begin{array}{l}\text { GARE } \\
\text { TTGGATAAACAGAGTAGAGATAGCGCCCACCATGGCACCTATAAGGAGAGAAATGTCATATATAGTAGTAGTACTCCTCCTC } \\
\text { WUN-mot if }\end{array}$ & -2097 \\
\hline 2096 & ATGTTGTTATATCATTAAGAAAGGCTTTGATAGTTGAATACCATACCACATCAGGAGTCTTGAGATAGATGAACAAGACG & -2017 \\
\hline 2016 & TGGACAGATTGGCAATGTAGAGAACACCAAAAAGAATCTGCACACAACATCAAACGAACGATTTGTTAAAATGCAGAGG & 37 \\
\hline 1936 & CGAGAAAGAGAGAGTCAATTTGAAGGTACCTGAGCAGGGTAGACTGCTCCCCCAGTCAAATTCTGGACAC & 5 \\
\hline 1856 & CATAGAGGAAACCAGCCGGATAAACCAAAGGCCCTGTGTCGCCTTTCAAGCTCCCGTAG & -1777 \\
\hline 1776 & ССАCTAACCTGCCCCCATTTTGTAAAATTTCACCAAGAGAGTTGACTTTGTATATTCTACTGGATGTAATGCAAACCTGT & 97 \\
\hline-1696 & ACATGTACGCATCCCAATCGATCTTCGTGTCTGTTCAGTAAATTT: & 1617 \\
\hline 1616 & TTGCACATTTCGAAATTAAAAAAAAACGTAGGAAATTAGATCTGACATGGAACATAGGCGATGATAAG & 1537 \\
\hline-1536 & TGCATCAGCCACGA' & 14 \\
\hline 1456 & AATCGCCCCAGICGT & -1377 \\
\hline 1376 & $\begin{array}{l}\text { ACAACTAACTCTTAGTTTTAGCCCATAGTAAAGCCC } \\
\text { ABRE } \quad \text { Skn-1_m }\end{array}$ & \\
\hline 296 & TATCCAAAACAAAACAATTTTATTTCCACGTGTACCACGTCTGT & -1217 \\
\hline 216 & $\begin{array}{l}\text { GCN4_mot if } \\
\text { ABRE /CE3 } \\
\text { GTCAACTACCCGCCGCGTGTCTCTCCAACACTCTTCTTCTTTTATAAACTTGAGCTCCTC }\end{array}$ & -1137 \\
\hline 136 & $\overline{5}$ & -10 \\
\hline 1056 & $\begin{array}{l}\text { GCCGCTATGGCTGAGACGGAGAGGAAACAAGCCGACGACAACAACAACAACAAAGGCAAACGCGAT } \\
\text { TGACGr-mot if }\end{array}$ & -977 \\
\hline-976 & GGCTAAACGCGGTCTCAAATCCCTGACGTTAGCTGT & -897 \\
\hline 896 & GGAATCAGGCTCGGTCCTCGTCGTGGGTCCTTCACCTCATGCGTCTCGCCTCGAGCGGTCTGATGGGCTTGGCTG & -817 \\
\hline-816 & $\begin{array}{l}\text { CTCGTATGGGTCGACGCTGGGTTCCACAAGAAGCCCAACGCTCTGTATCTTTACTTGGCTCAGTTTGTGCTTTGTTTGAC } \\
\begin{array}{l}\text { ABRE } \\
\text { GCN4_mot if }\end{array}\end{array}$ & -737 \\
\hline-736 & TACGTGCATGGTCGGGTCGGGACTAGCAGGGCTTGCAGTGTGCTTGTGTCAGTCTGCGGCCTTGTTCO & -657 \\
\hline-656 & CCTTTAATGAGATCAGTCCGGTCGCTGGTAATATGGTAAAGCCGTGTTTGGCTTTTGCT & -577 \\
\hline-576 & GTAAAGCTCGCAATCGCGTGAGATCTATAAACAGTATAAAGCCAACGATAGATGTGATAGTCTCTGAGAATGTTGTGGAG & -497 \\
\hline-496 & TCGTAATTAATCGTTTTTTTTTACAAGTGTTTTGTGACAATATACGTAATTAATTATCTGTTAGTATGTGTCCATTGGGC & -417 \\
\hline-416 & $\begin{array}{l}\text { TTTAATATATATCAAGATTGACAAAATAAAAGTGGCTACATTTGAGTTTAGGGTCCTGATTATTGCAGACTTATTGGCCG } \\
\text { CAAT-box }\end{array}$ & -337 \\
\hline-336 & 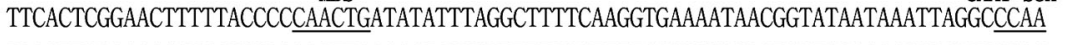 & -257 \\
\hline-256 & $\begin{array}{l}\text { TAAGTTCGAAGACGTGATGGAAAAGTTAGGGCTATTCTTTTTGCATTTTCCCTCGAGACTCGTGCCAGACGGAATGGGAG } \\
\text { ABRE }\end{array}$ & -177 \\
\hline-176 & $\begin{array}{l}\text { TGCTTATCTTGCAACAATCTCGACCCATGCCCTGACACGRGETGTGAGCGGTCCATTAGCATCAGTACACGTGTCCCAGC } \\
\text { MBS }\end{array}$ & -97 \\
\hline-96 & GGTCACTCTTCGCTTACAATCCTTGTCGTCTGCAGATCTTGTATTCAGTCCTTCTATTTAAAGAGTAAATTCAATCGGAA & -17 \\
\hline & CGAGTAGGATTTGAGAATGGCTGAAGATGAGATTATAGTC & \\
\hline
\end{tabular}

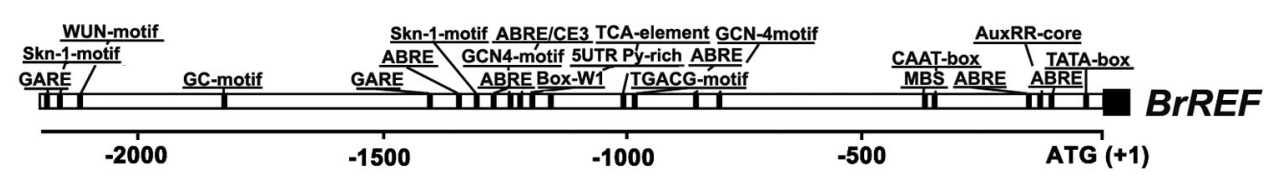

Fig. 7. Schematic representation of putative cis-acting elements in the BrREF promoter sequence. Numbering is based on the distance from the 5 '-end nucleotide sequence $(+1)$ of the open reading frame. The relative positions of potential cis-acting elements mentioned in the text are shown. 
expression mechanism of the $B r R E F$ gene, the $2.2 \mathrm{~kb}$ promoter region was isolated and analyzed in this study. The BrREF promoter-GUS construct was stably transformed into Arabidopsis and B. napus to examine whether the function of this promoter is conserved between different species. In transgenic Arabidopsis, histochemical analysis showed strong GUS activity in the cotyledons and hypocotyls of germinating seeds and during seedling growth, but, GUS expression was almost absent during the mature plant stages (Fig. 4A). In addition, GUS activity in reproductive stages was observed only in the immature styles, septums, and anthers of flower buds (Fig. 5C). In transgenic $B$. napus, the similar expression of GUS was observed in seedlings and in immature anther, but GUS expression in the anthers and stigma showed spatial and temporal differences (Fig. 4B and 5C). These results indicated that the function of $B r R E F$ promoter was presumably conserved in different species. These results were in agreement with the RT-PCR results; the pattern of GUS expression was strikingly similar to the pattern of BrREF and GUS expression during seedling and plant growth (Fig. 3 and 6). These findings suggest that the regulatory mechanisms controlling gene expression in specific tissues and during development in B. rapa are similar to those in Arabidopsis and B. napus. It is possible that the BrREF promoter may be regulated by similar regulatory factors and cis-elements in specific tissues and during development in plants (Saha et al. 2007; Yi et al. 2010). However, the different expression patterns in the other reproductive organs of transgenic Arabidopsis and B. napus plants may reflect differences in the specific roles of the gene in particular organs of different plant species and a combination of specific transcriptional regulatory elements that uniquely control their expression in different species (Yi et al. 2010).

In promoter regions, short consensus DNA sequences, cis-acting elements, serve as binding sites for transcription factors to initiate transcription and modulate complex gene regulation processes by combinatorial interaction (Saha et al. 2007). To understand the regulatory mechanisms that control $B r R E F$ gene expression, we analyzed the promoter sequences of the BrREF gene (Fig. 7). This analysis revealed that the potential regulatory elements associated with growth and hormone responses were identified in the promoter region of $B r R E F$ gene, including ABRE, AuxRRcore, CE3, 5'UTR-Py-rich, GCN4-motif, Skn-1 motif, and GARE. 5'UTR-Py-rich stretch controls the high transcription levels of gene expression in seed-specific promoter (Fang et al. 2013). Skn-1 motif is required for high levels of endosperm expression in cooperative interaction with other motifs, such as a GCN4-motif (Washida et al. 1999). GARE is essential for GA activation and sugar insensitivity in endosperms (Chen et al. 2006). Both motifs were found in $B r R E F$ promoter region and may modulate gene expression in developmental series of seedlings. Therefore, the presence of various elements suggests that target gene expression may be regulated by various cis-acting regulatory elements within the BrREF promoter and by the corresponding trans-acting factors and may be controlled by complex regulatory mechanisms that respond to developmental and environmental cues (Hong and Hwang 2009; Hwang et al. 2009). Although our data do not indicate whether the putative cis-acting elements influence gene expression directly or indirectly, our results demonstrate that the 5' upstream region of the $B r R E F$ gene can drive transgene expression specifically during seedling growth and in floral organs of transgenic plants, and could be used to determine the biological role and function of the $B r R E F$ gene and to produce target protein in specific tissues and growth stages in various plants.

In conclusion, we have identified and characterized a $R E F$ gene and its promoter region in B. rapa. To date, this is the first report of the isolation and characterization of a $R E F$ gene from B. rapa. We showed that the BrREF promoter region can drive specific spatial and temporal gene expression during plant growth and development in seedlings and floral organs in both Arabidopsis and $B$. napus, suggesting that the BrREF promoter may confer similar regulation of gene expression in heterologous transgenic plants. The specific spatial and temporal patterns of gene expression induced by the $B r R E F$ promoter during seedling growth and in floral organs could serve as a useful tool for the precise transcriptional regulation of tissuespecific and developmental expression of foreign genes in various plants. 


\section{ACKNOWLEDGMENTS}

This work was supported by a grant from the National Academy of Agricultural Science (PJ008616), the Rural Development Administration, Republic of Korea. J.K. Hong was supported by a 2014 Post Doctoral Course Program from the National Academy of Agricultural Science, Rural Development Administration, Republic of Korea.

\section{REFERENCES}

Burge CB, Karlin S. 1998. Finding the genes in genomic DNA. Curr. Opin. Struct. Biol. 238: 346-354.

Chen P-W, Chiang C-M, Tseng T-H, Yua S-M. 2006. Interaction between rice MYBGA and the gibberellin response element controls tissue-specific sugar sensitivity of a-amylase genes. Plant Cell 18: 2326-2340.

Dennis MS, Henzel WJ, Bell J, Kohr W, Light DR. 1989. Amino acid sequence of rubber elongation factor protein associated with rubber particles in Hevea latex. J. Biol. Chem. 264: 18618-18626.

Dennis MS, Light DR. 1989. Rubber elongation factor from Hevea brasiliensis. Identification, characterization and role in rubber biosynthesis. J. Biol. Chem. 264: 1860818617.

Desfeux C, Clough SJ, Bent AF. 2000. Female reproductive tissues are the primary target of Agrobacterium-mediated transformation by the Arabidopsis floral-dip method. Plant Physiol. 123: 895-904.

Fang W, Zhang Y, Zhou L, Wang W, Li X. 2013. Isolation and characterization of Histone1 gene and its promoter from tea plant (Camellia sinensis). Mol. Biol. Rep. 40: 3641-3648.

Han K-H, Shin DH, Yang J, Kim IJ, Oh SK, Chow K-S. 2000. Genes expressed in the latex of Hevea brasiliensis. Tree Physiol. 20: 503-510.

Hellens RP, Edwards EA, Leyland NR, Bean S, Mullineaux PM. 2000. pGreen: a versatile and flexible binary Ti vector for Agrobacterium-mediated plant transformation. Plant Mol. Biol. 42: 819-832.

Higo K, Ugawa Y, Iwamoto M, Korenaga T. 1999. Plant cis-acting regulatory DNA elements (PLACE) database. Nucleic Acids Res. 27: 297-300.
Holsters M, de Walaele D, Depicker A, Messens E, Van Montagu M, Schell J. 1978. Transfection and transformation of Agrobacterium tumefaciens. Mol. Gen. Genet. 163: 181-187.

Hong JK, Hwang BK. 2009. The promoter of the pepper pathogen-induced membrane protein gene CaPIMP1 mediates environmental stress responses in plants. Planta 229: 249-259.

Hong JK, Kim JA, Kim JS, Lee SI, Koo BS, Lee Y-H. 2012. Overexpression of Brassica rapa SHI-RELATEDSEQUENCE genes suppresses growth and development in Arabidopsis thaliana. Biotechnol. Lett. 34: 1561-1569.

Hong JK, Kim JS, Kim JA, Lee SI, Lim MH, Park BS, Lee YH. 2010. Identification and characterization of SHI family genes from Brassica rapa L. ssp. Pekinensis. Genes Genom. 32: 309-317.

Hong JK, Kim S-Y, Kim K-S, Kwon S-J, Kim JS, Kim JA, Lee SI, Lee Y-H. 2013. Overexpression of a Brassica rapa MADS-box gene, BrAGL20, induces early flowering time phenotypes in Brassica napus. Plant Biotechnol. Rep. 7: 231-237.

Hong JK, Kim S-Y, Kim JS, Kim JA, Park B-S, Lee Y-H. 2011. Promoters of three Brassica rapa FLOWERING LOCUS C differentially regulate gene expression during growth and development in Arabidopsis. Genes Genom. 33: $75-82$.

Hwang JE, Hong JK, Je JH, Lee KO, Kim DY, Lee SY, Lim CO. 2009. Regulation of seed germination and seedling growth by an Arabidopsis phytocystatin isoform, AtCYS6. Plant Cell Rep. 28: 1623-1632.

Jefferson RA, Kavanagh TA, Bevan MW. 1987. GUS fusions: $\beta$-glucuronidase as a sensitive and versatile gene fusion marker in higher plants. EMBO J. 6: 3901-3907.

Kim S-Y, Park B-S, Kwon S-J, Kim JS, Lim M-H, Park Y-D, Kim DY, Suh S-C, Jin YM, Ahn JH, Lee Y-H. 2007. Delayed flowering time in Arabidopsis and Brassica rapa by the overexpression of FLOWERING LOCUS C (FLC) homologs isolated from Chinese cabbage (Brassica rapa L. ssp. pekinensis). Plant Cell Rep. 26: 327-336.

Kush A, Goyvaerts E, Chye ML, Chua ML. 1990. Laticifer specific gene expression in Hevea brasiliensis (rubber tree). Proc. Natl. Acad. Sci. USA 87: 1787-1790.

Liang YC, Bae H-J, Kang S-H, Lee T, Kim MG, Kim YM and Ha S-H. 2009. The Arabidopsis beta-carotene hydroxylase gene promoter for a strong constitutive expression of 
transgene. Plant Biotechnol. Rep. 3: 325-331.

Liang YC, Jeon Y-A, Lim S-H, Kim JK, Lee J-Y, Kim Y-M, Lee Y-H, Ha S-H. 2011. Vascular-specific activity of the Arabidopsis carotenoid cleavage dioxygenase 7 gene promoter. Plant Cell Rep. 30: 973-980.

Lukashin AV, Borodovsky M. 1998. GeneMark.hmm:new solutions for gene finding. Nucleic Acids Res. 26: $1107-$ 1115.

Murashige T, Skoog F. 1962. A revised medium for rapid growth and bioassays with tobacco tissue cultures. Physiol. Plant 15: 473-497.

Priya P, Venkatachalam P, Thulaseedharan A. 2006. Molecular cloning and characterization of the rubber elongation factor gene and its promoter sequence from rubber tree (Hevea brasiliensis): A gene involved in rubber biosynthesis. Plant Sci. 171: 470-480.

Priya P, Venkatachalam P, Thulaseedharan A. 2007. Differential expression pattern of rubber elongation factor (REF) mRNA transcripts from high and low yielding clones of rubber tree (Hevea brasiliensis Muell. Arg.). Plant Cell Rep. 26: 1833-1838.

Rombauts SS, Déhais P, Van montaqu M, Rouzé P. 1999. PlantCARE, a plant cis-acting regulatory element database. Nucleic Acids Res. 27:295-296.

Saha D, Prasad AM, Sujatha TP, Kumar V, Jain PK, Bhat SR, Srinivasan R. 2007. In silico analysis of the Lateral
Organ Junction (LOJ) gene and promoter of Arabidopsis thaliana. In Silico Biol. 7: 7-19.

Washida H, Wu C-Y, Suzuki A, Yamanouchi U, Akihama T, Harada K, Takaiwa F. 1999. Identification of cis-regulatory elements required for endosperm expression of the rice storage protein glutelin gene GluB-1. Plant Mol. Biol. 40: $1-12$.

Yang KA, Lim CJ, Hong JK, Park CY, Cheong YH, Chung YS, Lee KO, Lee SY, Cho MJ, Lim CO. 2006. Identification of cell wall genes modified by a permissive high temperature in Chinese cabbage. Plant Sci. 171: 175-182.

Yao K, Lockhart KM, Kalanack JJ. 2005. Cloning of dehydrin coding sequences from Brassica juncea and Brassica napus and their low temperature-inducible expression in germinating seeds. Plant Physiol. Biochem. 43: 83-89.

Yi J, Derynck MR, Chen L, Dhaubhadel S. 2010. Differential expression of $C H S 7$ and $C H S 8$ genes in soybean. Planta 231: 741-753.

Zang Y-X, Kim HU, Kim JA, Lim M-H, Jin M, Lee SC, Kwon S-J, Lee S-I, Hong JK, Park T-H, Mun J-H, Seol Y-J, Hong S-B, Park B-S. 2009. Genome-wide identification of glucosinolate synthesis genes in Brassica rapa. FEBS J. 276: 3559-3574. 


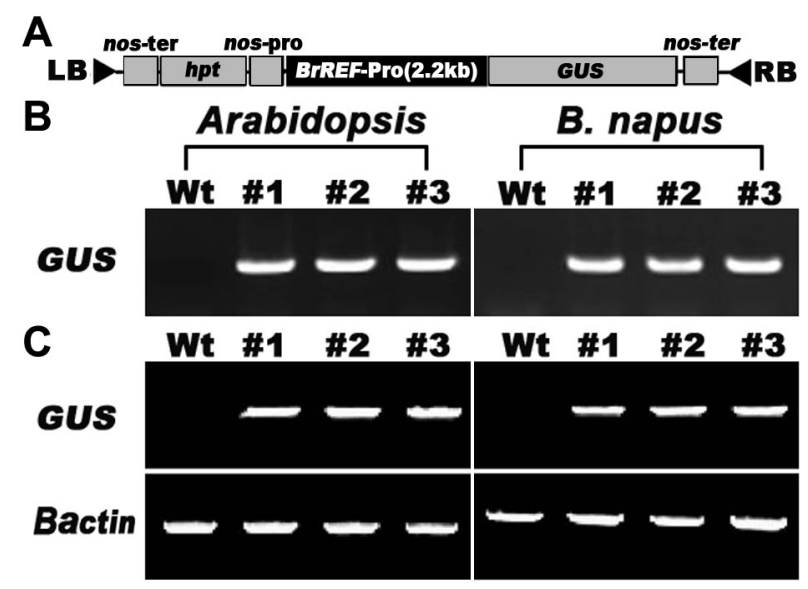

Supplemental Fig. 1. GUS gene expression in transgenic Arabidopsis and B. napus. (A) Structure of the BrREF promoterGUS construct used for Arabidopsis and B. napus transformation. The hygromycin phosphotransferase gene ( $h p t$ ), with a nopaline synthase gene promoter (nos-pro) and 3' terminator (nos-ter), served as the selectable marker for Arabidopsis and B. napus transformation. GUS expression was driven by the BrREF promoter. Left and right T-DNA borders are indicated by LB and RB, respectively. (B) PCR analysis of genomic DNA isolated from control and transgenic plants. The presence of the GUS gene was verified by PCR amplification using gene-specific primers. (C) Semi-quantitative RT-PCR to detect GUS gene expression in transgenic plants. Bactin gene expression was used as a quantitative control. Lanes: Wt, untransformed wild-type; lanes \#1-3, selected transgenic lines 1, 2, and 3. 